\title{
Glycoconjugates, promising subjects for medical science
}

\author{
Munehiro Nakata* \\ *Co-Editor-in-Chief, Drug Discoveries \& Therapeutic; \\ Department of Applied Biochemistry, Tokai University School of Engineering, Kanagawa, Japan.
}

\begin{abstract}
A long period of studies on carbohydrates such as glycans and carbohydrate moieties of glycoconjugates has brought us a lot of understandings on various biological phenomena in the molecular level. Many of biological phenomena, such as cell-cell recognition, clearance of glycoproteins in blood, infection of pathogens, and initiation of innate immunity, are now recognized as those triggered via carbohydrate recognition. These studies have developed not only understandings on biological rolls of carbohydrate moieties of glycoconjugates but also established glycotechnology including glycan analyses, modification of oligosaccharide structure in glycoconjugates, and creation of novel glycosylated substances. Nowadays, glycotechnology and knowledge on carbohydrate-related biological phenomena are applied in a wide range of fields, especially medical science.

This issue carries a series of articles including reviews on immunomodulation by polysaccharides and advanced technologies for structural analysis of glycans as well as some original studies on carbohydrate-related biological functions aiming medical use. These biochemical and/or physiological understandings on carbohydrates or glycoconjugates are expected to progress new approaches to overcome diseases. Finally, I express my gratitude to Dr. Fengshan Wang, Shandong University, Ji'nan, China, for his active management of these articles.
\end{abstract}

(Received April 19, 2015) 